\title{
Explosions of Cylindrical Pressure Vessels Subjected to Fire: Probabilistic Prediction of a Number of Fragments
}

\author{
Egidijus Rytas VAIDOGAS \\ Vilnius Gediminas Technical University, Sauletekioal.11,10223Vilnius, Lithuania, E-mail: egidijus.vaidogas@vgtu.lt \\ crossref http://dx.doi.org/10.5755/j02.mech.29014
}

\section{Introduction}

Cylindrical steel pressure vessels are widely used in various branches of industry. Most of them are parts of stationary equipment operated in industrial facilities. However, a fairly large population of such vessels is used for a transportation of hazardous materials by road and rail. A major portion of such materials are commercial energetic hydrocarbons. A transportation of hazardous materials poses the risk of major accidents. Pressurised hydrocarbons shipped by road or rail are especially prone to such accidents [1].

The most severe transportation accident, in which cylindrical steel vessels can be involved, is the so-called boiling liquid expanding vapour explosion (BLEVE) [2-4]. This explosion occurs as a violent disintegration of a vessel exposed to external heating by a fire. It generates fragments that are the furthest reaching hazard from a BLEVE [5]. Blast and fireball with an intense thermal radiation are also generated during the catastrophic vessel failure. Clearly, a broad spectrum of pressure vessels includes explosions triggered off by very different phenomena. However, this study will be limited by BLEVEs of unconstrained transportation vessels for brevity sake.

An accurate forecasting of fragment numbers and fragmentation patterns exclusively by means of deterministic mechanical analysis or metallurgical examination is not possible to date. Metallurgical analysis was applied once to a post mortem investigation of a transportation vessel after a BLEVE accident [6]. Mechanical analysis has been limited mainly to a FEM assessment of heat induced stresses and deformations of vessels exposed to fires [7]. For such vessels, FEM was also applied in combination with the computational fluid dynamics (CFD) [8]. The field of FEM and CFD applications to the case of externally heated vessels is now in the course of rapid development. However, a BLEVE is a process that begins with fire induced initial local rupture of vessel wall and ends with formation of circumferential cracks and vessel fragmentation [9]. To the best of our knowledge, a reliable and accurate prediction of this process solely by means of FEM and CFD methods is hardly possible at the present time. One of the reasons for that may be the fact that the scenario of vessel fracture depends on random factors: uncertainties related to fire impingement, vessel corrosion, defects, damages due to external impacts [10]. These uncertainties could be taken into account by applying methods of reliability analysis and probabilistic risk assessment (PRA). However, a combined application of probabilistic methods and deterministic mechanical analysis to the case of BLEVE still remains an unresolved problem.

Hazards and consequences of BLEVEs and vessel explosions of other types are natural subjects of PRA. Unlike the classical, deterministic mechanical analysis, PRA is focussed on quantifying uncertainties, related to fragment numbers, fragmentation patterns, ejection and projection of fragments $[11,12]$. The first step in assessing the event sequence starting with vessel fragmentation and ending with fragment impact on a target is a prediction of the number of fragments and a closely related prediction of fragmentation pattern.

Despite seemingly chaotic and unforeseeable nature of pressure vessels bursts, the fragmentation of vessels that undergo BLEVEs brinks out some regularity in terms of fragment numbers and fragmentation patters [13, 14]. This relatively weak regularity allows to approximately predict the number of fragments generated by BLEVEs. However, the prediction will face the need to quantify uncertainties related to the fragmentation. PRA provides a useful format for approaching this problem through the use of currently available accident data.

The present study is devoted to a prediction of the number of fragments from bursts of horizontally oriented pressure vessels. The prediction relies on relatively scarce data on past accidents and is expressed in terms of probabilities of individual fragment numbers. The study demonstrates how to apply the Bayesian analysis to assessing uncertainties in fragment number probabilities (epistemic or reducible uncertainties in terms of PRA). Results of this study can contribute to an improvement of PRA related to pressure vessels and ultimately a safer use of these vessels.

\section{Data on fragment numbers}

A prediction of a fragmentation (failure) pattern of a vessel is the first step in estimating the probability of fragment impact on a potential target and damage to this target (Fig. 2). The fragmentation pattern will affect most of the factors that influence the damage probability and severity and, first of all, the number of primary fragments (parts of the vessel body).

Bursting of an unconstrained, nonstationary pressure vessel used for shipment of liquefied gas, say, will produce either one fragment (ruptured body of the vessel with no detached parts) or eject two or more fragments (Fig. 1) At the same time, data on past BLEVE accidents indicates that the number of fragments, $n_{f r g}$, of transportation vessels made of ductile steel will not exceed the value of nine [14]. In addition, the relative frequencies (proportions) of fragment numbers, $\operatorname{fr}\left(n_{f r g}\right)\left(n_{f r g}=1,2, \ldots, 9\right)$, counted from the past accidents decrease rapidly with increasing $n_{f r g}$ (Table 1). In addition, the frequencies $f r(2)$ and $f r(3)$ prevail among the remaining ones. Thus a prediction of fragment 
masses $m_{f r g, j}$ and shapes and, subsequently, the ejection velocities should be focussed mainly on the fragmentation patters shown in Fig. 1, as long as safety of potential targets is of concern.

a) fragments are not ejected, no detached parts of the vessel

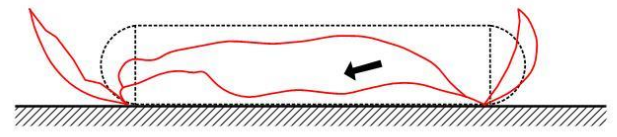

b) two fragments (oblong end-caps) are ejected

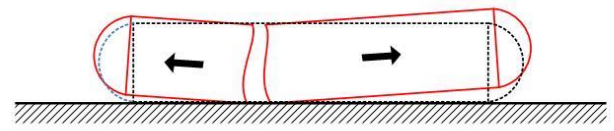

c) three of more fragments (end-caps and cylindrical ring) are ejected

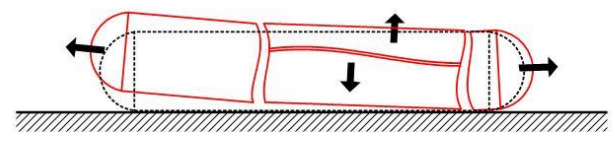

Fig. 1 Probable fragmentation patterns of an unconstrained vessel (e.g., vessel of a road or railway tanker involved in a traffic accident)

Table 1

Data on fragmentation of cylindrical vessels in the course of BLEVEs [14]

\begin{tabular}{|c|c|c|}
\hline$n_{f r g}$ & Count of BLEVEs & Frequency $f r\left(n_{f r g}\right)$ \\
\hline 1 & 50 & 0.1931 \\
2 & 98 & 0.3784 \\
3 & 78 & 0.3012 \\
4 & 24 & 0.0927 \\
5 & 3 & 0.0116 \\
6 & 2 & 0.0077 \\
7 & 3 & 0.0116 \\
8 & 0 & 0.0000 \\
9 & 1 & 0.0039 \\
\hline Total: & 259 & 1.0 \\
\hline
\end{tabular}

In general, the uncertainty related to the potential number of fragments can be expressed by a random variable $N_{f r g}$ with a set of countably infinite values $n_{f r g}$. The variable $N_{f r g}$ should obey a certain discrete probability distribution, the masses of which $P\left(N_{f r g}=n_{f r g}\right)\left(n_{f r g}=1,2, \ldots\right)$, are probabilities of generating a given number of fragments. The probability masses $P\left(N_{f r g}=n_{f r g}\right)$ can be fitted to the data (a set of values of $N_{f r g}$ counted in post mortem investigations of accidents) in a standard, formal way. For instance, if goodness of fit tests reveal that $N_{f r g}$ can be described by a Poisson distribution, the average number of fragments per accident, $\bar{n}_{f r g}$, would suffice to specify this model.

From the standpoint of a fragment impact assessment, a formal fitting of a discrete distribution to the data on values of $N_{f r g}$ can be misleading. Firstly, the probability $P\left(N_{f r g}=1\right)$ expresses the chance of a relatively safe scenario of vessel bursting. The random event $N_{f r g}=1$ represents an accident scenario, in which no detached parts of the vessel are ejected and the remnant of the vessel remains close to its initial rest position.

The data was presented in Table 1 was lumped together by Sun et al. [14] from various sources and covers explosions of stationary and unconstrained vessels. A detailed description of this data set is not provided. The 50 out of 259 BLEVEs with only one fragment (an occurrence of the event $\left.N_{f r g}=1\right)$ should be related to bursts without ejection of fragments (Fig. 1, a). One can guess that these 50 accidents might also involve bursts of vessels in which only one fragment was ejected and the rest of vessel remained on supporting structure (Fig. 2, a). A fragmentation of an unconstrained vessel in an ejected part and a remainder that stays in place is hardly possible. The minimum number of projectiles for such vessels should be two (Fig. 2, b).

a) constrained vessel: one projectile is detached from the vessel body

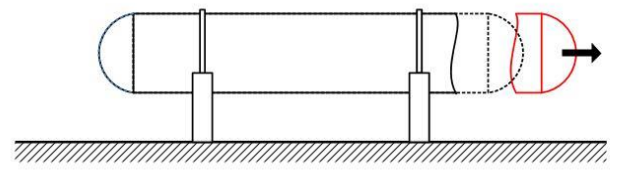

b) unconstrained vessel: at least two projectiles are ejected

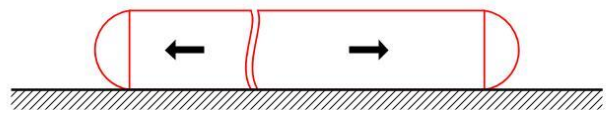

Fig. 2 Minimal number of hazardous fragments (oblong end-caps) in case of constrained and unconstrained vessel

Data on BLEVE accidents show that the counts of vessel disintegrations into more than four fragments are very small and so the probability $P\left(N_{f r g} \geq 5\right)$ represents unlikely accident scenarios (Table 1 ). In addition, bursting of vessel into a large number of fragments will be relatively safe because masses of fragments and so the damaging potential will be small. Consequently, only the probabilities $P\left(N_{f r g}=2\right), P\left(N_{f r g}=3\right)$ and $P\left(N_{f r g}=4\right)$ will be of interest to a fragment impact assessment.

\section{Bayesian estimation of fragment numbers by means of Poisson-gamma model}

An estimation of the probabilities of fragment numbers exceeding one can be based on 209 accidents in which at least two fragments were generated (Table 2). Relative frequencies of BLEVE accidents with two to nine fragments are presented in the third of Table 2. The dominant numbers of fragments were two and three ( $84.2 \%$ of cases). Four fragments were generated only in $11,5 \%$ of cases. Five to nine fragments were encountered only in $4.3 \%$ of cases.

Until now, a probability distribution of the fragment number $N_{f r g}$ was fitted in the classical statistical (Fisherian) format by means of the maximum entropy method $[13,15,16]$. However, this method yields a continuous exponential distribution with three parameters which are difficult to handle in the Bayesian format. A single-parameter discrete probability distribution of $N_{f r g}$ is preferable to carrying out Bayesian updating.

The distribution of the relative frequencies $f r\left(n_{f r g}\right)$ given in Table 2 can be reasonably approximated by displaced standard discrete distributions. If $N_{f r g}$ is a random variable representing the number of fragments, the distribution of $N_{f r g}$ given that $N_{f r g} \geq 2$, could be such that the random variable:

$$
N_{0}=N_{f r g}-2
$$

has approximately a standard single-parameter probability distribution. In the case of a Bayesian analysis, the most 
problematic part will be a development of an appropriate prior for this parameter.

Table 2

Counts and frequencies related to disintegration of cylindrical vessels into at least two fragments (obtained from Table 1)

\begin{tabular}{|c|c|c|c|}
\hline $\begin{array}{c}\text { No of frag- } \\
\text { ments } n_{f r g}\end{array}$ & $\begin{array}{c}\text { Count of } \\
\text { BLEVEs }\end{array}$ & $\begin{array}{c}\text { Fre- } \\
\text { quency } \\
f r\left(n_{f r g}\right)\end{array}$ & $\begin{array}{c}\text { Displaced no of frag- } \\
\text { ments } n_{0}=n_{f r g}-2\end{array}$ \\
\hline 1 & - & - & - \\
2 & 98 & 0.4689 & 0 \\
3 & 78 & 0.3732 & 1 \\
4 & 24 & 0.1148 & 2 \\
5 & 3 & 0.0144 & 3 \\
6 & 2 & 0.0096 & 4 \\
7 & 3 & 0.0144 & 5 \\
8 & 0 & 0.0000 & 6 \\
9 & 1 & 0.0048 & 7 \\
\hline Total: & 209 & 1.0 & - \\
\hline
\end{tabular}

The natural candidate for describing $N_{0}$ is the Poisson distribution. If we denote the probability mass function of this distribution by $p\left(n_{0} \mid \lambda_{0}\right)\left(n_{0}=0,1,2, \ldots\right)$, the parameter $\lambda_{0}$ will express the displaced average number of fragments per one BLEVE with two or more fragments. Value of $\lambda_{0}$ can be estimated by solving the minimisation problem:

$$
\hat{\lambda}=\min _{\lambda}\left(\sum_{n=2}^{9}\left|f r(n)-p\left(n-2 \mid \lambda_{0}\right)\right|\right)
$$

where: $\hat{\lambda}$ denotes and estimate of estimates of $\lambda_{0}$. For the frequencies $f r\left(n_{f r g}\right)$ given in Table 2, Eq. (2) yields the following estimate $\hat{\lambda}=0.758$. The pairs $f r\left(n_{0}\right)$ and $p\left(n_{0} \mid 0.758\right)$ are depicted in Fig. 3.

Fitting a discrete probability distribution to the frequencies $f r\left(n_{0}\right)$ does not bring much of practical information, no matter how good theoretical model matches empirical distribution. However, the suitability of Poisson distribution for describing the displaced fragment number $N_{0}$ opens up a possibility to apply the standard Bayesian analysis to an assessment of the population parameter $\lambda_{0}$ and prediction of individual fragment numbers. In PRA, the Poisson distribution applied this prediction is called" the model of the world" [17].

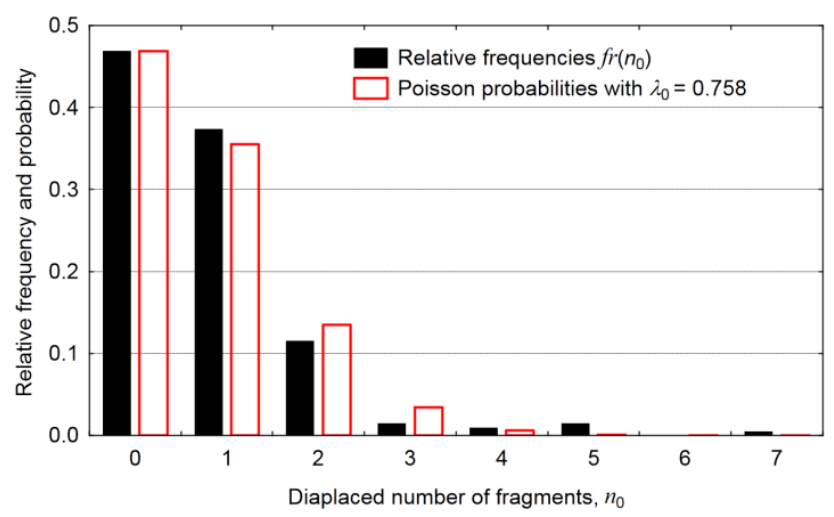

Fig. 3 Poisson probabilities $p\left(n_{0} \mid 0.758\right)$ and empirical frequencies $f r\left(n_{0}\right)$ vs the displaced fragment number $n_{0}$ $\left(n_{0}=0,1,2, \ldots, 7\right)$
In the case of the Poisson distribution, a gamma prior for the unknown mean $\lambda_{0}$ of $N_{0}$ leads to a conjugate analysis. The new data can be gained from BLEVEs of unconstrained vessels which will occur in the future or past BLEVE accidents which previously were not included into the analysis.

The new data used for updating the gamma prior may has the form

$$
\boldsymbol{n}_{0}^{\prime}=\left\{n_{0 m}^{\prime}, m=1,2, \ldots, n_{a}^{\prime}\right\},
$$

where: $n_{0 m}^{\prime}$ is the displaced number of primary fragments counted in the BLEVE accident number $m$ and $n_{a}^{\prime}$ is the number of accidents $\left(n_{a}^{\prime} \geq 1\right)$. Here and in what follows, the apostrophe symbol denotes information related to new data and posterior distribution. New data used in PRA applications has the standard form $\{r$ failures in time $t\}$ with the ratio $r / t$ meaning the average number of failures per unit time. Therefore, the information expressed by Eq. (3) must be interpreted as $\left\{n_{t o t}^{\prime}\right.$ fragments in $n_{a}^{\prime}$ accidents $\}$, where $n_{\text {tot }}^{\prime}$ is the total number of fragments counted in $n_{a}^{\prime}$ accidents, that is:

$$
n_{t o t}^{\prime}=\sum_{m=1}^{n_{a}^{\prime}} n_{0 m}^{\prime} .
$$

Then the proportion $n_{t o t}^{\prime} / n_{a}^{\prime}$ means the average number of fragments counted in the accidents used to compile $\boldsymbol{n}_{0}^{\prime}$.

If a gamma prior $\operatorname{Gamma}(\alpha, \beta)$ is adopted for $\lambda_{0}$, then the posterior density of $\lambda_{0}$ will be again a gamma density with the parameters:

$$
\begin{aligned}
& \alpha^{\prime}=\alpha+n_{t o t}^{\prime}, \\
& \beta^{\prime}=\beta+n_{a}^{\prime} .
\end{aligned}
$$

As $n_{a}^{\prime}$ is the observed number of accidents, the parameter $\beta$ can be interpreted as a prior number of pseudoevents (pseudo-count of explosion accidents). Eq. (5a) implies that the shape parameter $\alpha$ must be interpreted as a pseudo-count of fragment numbers obtained in the prior pseudo-events (accidents). The analyst is not restricted to take the conjugate gamma prior. However, a Bayesian analysis with such a prior is very simple. The most problematic part of it will be a development of an appropriate gamma prior $\operatorname{Gamma}(\alpha, \beta)$.

The possibility to choose the prior $\operatorname{Gamma}(\alpha, \beta)$ ranges between noninformative and informative prior distributions. These two extreme forms of priors are diffuse and not suitable for updating with scarce data [17]. BLEVEs of horizontal pressure vessels are rare events even on the global scale. Therefore, it is very probable that the new data $\boldsymbol{n}_{0}^{\prime}$ gained in the foreseeable future will be scarce (contain a small number of components, $n_{a}^{\prime}$ ). Therefore we think that $\operatorname{Gamma}(\alpha, \beta)$ should be specified in the form of a constrained noninformative prior density (CNI prior) used in 
PRA for parameter estimation [18]. This density is also called the minimally informative prior. Such a prior is responsive to updates with scarce data.

The term "constrained" means that the prior is constrained to have a specified mean value $E\left[\Lambda_{0}\right]$, where $\Lambda_{0}$ is the random variable with the distribution $\operatorname{Gamma}(\alpha, \beta)$ used to model the epistemic uncertainty in the parameter $\lambda_{0}$ . The specification of $E\left[\Lambda_{0}\right]$ allows to incorporate minimum information into the prior $\operatorname{Gamma}(\alpha, \beta)$. The specified value of $E\left[\Lambda_{0}\right]$ can be the empirical average of displaced fragment numbers, $\bar{\lambda}_{0}$, counted in 209 accidents from Table 2, namely:

$$
E\left[\Lambda_{0}\right]=\bar{\lambda}_{0}=\sum_{n_{0}=0}^{7} n_{0} f r\left(n_{0}+2\right) .
$$

With the value $\bar{\lambda}_{0}$ of $E\left[\Lambda_{0}\right]$, the parameters of $\operatorname{Gamma}(\alpha, \beta)$ are specified as follows [19]:

$$
\begin{aligned}
& \alpha=0.5 . \\
& \beta=\frac{0.5}{\bar{\lambda}_{0}} .
\end{aligned}
$$

The parameters of the posterior distribution $\operatorname{Gamma}\left(\alpha^{\prime}, \beta^{\prime}\right)$ are of the form:

$$
\begin{aligned}
& \alpha^{\prime}=0.5+n_{t o t}^{\prime} . \\
& \beta^{\prime}=\frac{0.5}{\bar{\lambda}_{0}}+n_{a}^{\prime} .
\end{aligned}
$$

The posterior mean of the uncertain displaced Poisson intensity $\Lambda_{0}$ can be expressed as:

$$
E\left[\Lambda_{0} \mid n_{t o t}^{\prime}, n_{a}^{\prime}\right]=\frac{\alpha^{\prime}}{\beta^{\prime}}=\frac{\beta}{\beta+n_{a}^{\prime}} \frac{\alpha}{\beta}+\frac{n_{a}^{\prime}}{\beta+n_{a}^{\prime}} \frac{n_{t o t}^{\prime}}{n_{a}^{\prime}}
$$

where: $\alpha / \beta$ is the prior mean $E\left[\Lambda_{0}\right]$. The ratio $\alpha / \beta$ expresses the expected number of fragments per accident and $n_{t o t}^{\prime} / n_{a}^{\prime}$ is empirical proportion related to average fragment number. The posterior mean $E\left[\Lambda_{0} \mid n_{\text {tot }}^{\prime}, n_{a}^{\prime}\right]$ is a weighted average of $\alpha / \beta$ and $n_{t o t}^{\prime} / n_{a}^{\prime}$ with the weights $\beta /\left(\beta+n_{a}^{\prime}\right)$ and $n_{a}^{\prime} /\left(\beta+n_{a}^{\prime}\right)$, respectively.

If $\beta>n_{a}^{\prime}$, then then the prior $\operatorname{Gamma}(\alpha, \beta)$ will weigh more. In case that $\beta<n_{a}^{\prime}$, the data $\left(n_{t o t}^{\prime}, n_{a}^{\prime}\right)$ will weigh more. If $\beta=n_{a}^{\prime}$, prior and data will weight equally. Thus the parameter $\beta$ determines how long we need to observe vessel explosions until the posterior $\operatorname{Gamma}\left(\alpha^{\prime}, \beta^{\prime}\right)$ starts to move away from the prior $\operatorname{Gamma}(\alpha, \beta)$.

The transformation given by Eq. (1) is a liner one with the scale parameter equal to unity. Thus probability distributions and variances of the actual and displaced numbers of fragments, $N_{0}$ and $N_{f r g}$, are identical, whereas the mean values $E\left[N_{0}\right]$ and $E\left[N_{f r g}\right]$ differ by the shift parameter 2 . Therefore, the following simple relation holds:

$$
P\left(N_{f r g}=n_{f r g}\right)=P\left(N_{0}=n_{f r g}-2\right) \quad\left(n_{f r g}=2,3,4\right) .
$$

The probability mass function of the Poisson distribution allows to transform the uncertainty expressed by the random intensity $\Lambda_{0}$ into an epistemic uncertainty related to the fragment number probabilities $P\left(N_{f r g}=n_{f r g}\right)$. This uncertainty can be modelled by the random variables $\tilde{P}\left(n_{f r g}\right)$ defined as:

$$
\tilde{P}\left(n_{f r g}\right)=P\left(N_{f r g}=n_{f r g} \mid \Lambda_{0}\right)=\frac{\Lambda_{0}^{n_{f r g}-2}}{\left(n_{f r g}-2\right) !} \mathrm{e}^{-\Lambda_{0}} .
$$

The probability distribution of the random variables $\tilde{P}\left(n_{f r g}\right)$ can be estimated by means of the stochastic (Monte Carlo) simulation. It allows to propagate uncertainty expressed by $\Lambda_{0}$ to uncertainty in $P\left(N_{f r g}=n_{f r g}\right)$. In what follows, this propagation is illustrated by a numerical example.

\section{Example calculations}

The data given in Table 2 allows to calculate the weighted average of the displaced numbers of fragments, $n_{0}$, that range between 0 and 7 , namely,

$\bar{\lambda}_{0}=\sum_{n_{0}=0}^{7} n_{0} f r\left(n_{0}+2\right)=0 \times 0.4689+\ldots+7 \times 0.0048=0.789$.

This means that the weighted average of the actual fragment numbers is equal to 2.789 (see Eq. (1)). With the value 0.789 , the parameters of the prior $\operatorname{Gamma}(\alpha, \beta)$ are $\alpha=0.5$ and $\beta=0.5 / 0.789=0.634$.

Let the new actual information in this example be the numbers of fragments counted in 4 accidents with at least two fragments, namely, $\{3,2,2,4\}$. Then the new data suitable for updating and defined by Eq. (3) is $\boldsymbol{n}_{0}^{\prime}=$ $=\{1,0,0,2\}$ and $n_{a}^{\prime}=4$.

Thus the total displaced number of fragments in this data, $n_{t o t}^{\prime}$, is equal to 3 . With $n_{t o t}^{\prime}$ and $n_{a}^{\prime}$, the posterior distribution parameters are $\alpha^{\prime}=0.5+3=3.5$ and $\beta^{\prime}=$ $=0.634+4=4.63$

The density functions of the prior $\operatorname{Gamma}(0.5,0.634)$ and the posterior $\operatorname{Gamma}(3.5,4.66)$ are plotted in Fig. 4.

The weights given in Eq. (9) are $\frac{\beta}{\beta+n_{a}^{\prime}}=$ $=\frac{0.634}{0.634+4}=0.1368$ and $\frac{n_{a}^{\prime}}{\beta+n_{a}^{\prime}}=\frac{4}{0.634+4}=0.8632$.

This result together with the fact that the value of $n_{a}^{\prime}$ exceeded the value of $\beta$ more than six times indicate that the posterior shifted considerably away from the prior. This is revealed also by the graph given in Fig. 4 .

With the prior and posterior distributions $\operatorname{Gamma}(0.5,0.634)$ and $\operatorname{Gamma}(3.5,4.63)$ of the displaced Poisson intensity $\Lambda_{0}$ and the transformation given 
by Eq. (11), one can assess epistemic uncertainties in the probabilities $P\left(N_{f r g}=n_{f r g}\right) \quad\left(n_{f r g}=2,3,4\right)$. This can be done by applying the stochastic simulation to numerical transforming the distribution of $\Lambda_{0}$ into distributions of $\tilde{P}\left(n_{f r g}\right)$.

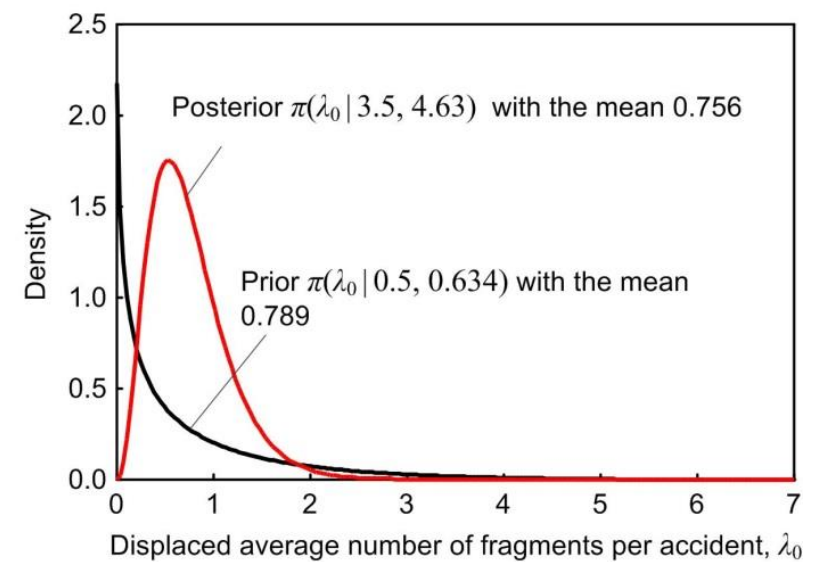

Fig. 4 Prior and posterior distributions for the displaced mean of the fragment number per accident, $\lambda_{0}$

The uncertainty transformation has been carried out for both prior and posterior distributions of $\Lambda_{0}$. Let the value of $\tilde{P}\left(n_{f r g}\right)$ computed in the simulation run $i$ by sampling from the prior be $p_{i}\left(n_{f r g}\right)$ and corresponding value obtained by sampling from the posterior be $p_{i}^{\prime}\left(n_{f r g}\right)$. These values were calculated as:

$$
\begin{aligned}
& p_{i}\left(n_{f r g}\right)=\frac{\left(\lambda_{0 i}\right)^{n_{f r g}-2}}{\left(n_{f r g}-2\right) !} \mathrm{e}^{-\lambda_{0 i},}, \\
& p_{i}^{\prime}\left(n_{f r g}\right)=\frac{\left(\lambda_{0 i}^{\prime}\right)^{n_{f r g}-2}}{\left(n_{f r g}-2\right) !} \mathrm{e}^{-\lambda_{0 i}^{\prime}},
\end{aligned}
$$

where: $\lambda_{0 i}$ and $\lambda_{0 i}^{\prime}$ are the values of $\Lambda_{0}$ sampled in the simulation run $i$ from $\operatorname{Gamma}(0.5,0.634)$ and Gamma $(3.5,4.63)$, respectively . A total of $n=10000$ simulation runs was used. The simulation produced two triplets of the samples related to the fragment numbers 2, 3 and 4:

$$
\begin{aligned}
& \boldsymbol{p}_{m}=\left\{p_{i}(m), i=1, \ldots, n\right\}(m=2,3,4), \\
& \boldsymbol{p}_{m}^{\prime}=\left\{p_{i}^{\prime}(m), i=1, \ldots, n\right\}(m=2,3,4) .
\end{aligned}
$$

Descriptive measures of these samples are given in Table 3. Histograms of the sample pairs $\left(\boldsymbol{p}_{m}, \boldsymbol{p}_{m}^{\prime}\right)(\mathrm{m}=2$, $3,4)$ are shown in Fig. 5.

Results expressed by Table 3 and Fig. 5 indicate that epistemic uncertainty in the fragment number probabilities $P\left(N_{f r g}=n_{f r g}\right)$ is large. The coefficients of variation (COVs) of the prior samples $\boldsymbol{p}_{m}$ range between $51 \%$ and $109 \%$. This can be explained by diffuseness of the prior distribution Gamma( $0.5,0.634)$. The COV of this distribution is equal to $141 \%$ and the $5^{\text {th }}$ to $95^{\text {th }}$ percentile credible interval is $(0.003,3.03)$. Thus the width of the credible interval exceeds by far the range of the interval of displaced fragment numbers considered in the analysis, namely, the interval $[0,2]$. The variability of the posterior samples $\boldsymbol{p}_{m}^{\prime}$ is smaller. COVs of $\boldsymbol{p}_{m}^{\prime}$ vary from $34 \%$ to $56 \%$. The COV of the posterior $\operatorname{Gamma}(3.5,4.63)$ is equal to $53.5 \%$, whereas the $90 \%$ credible interval is $(0.23,1.56)$. It is relatively narrow when compared to the interval $[0,2]$.

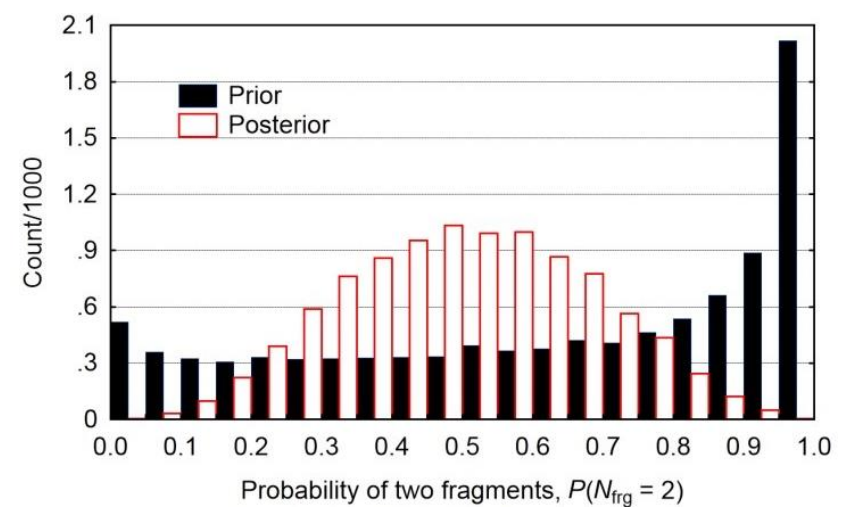

a

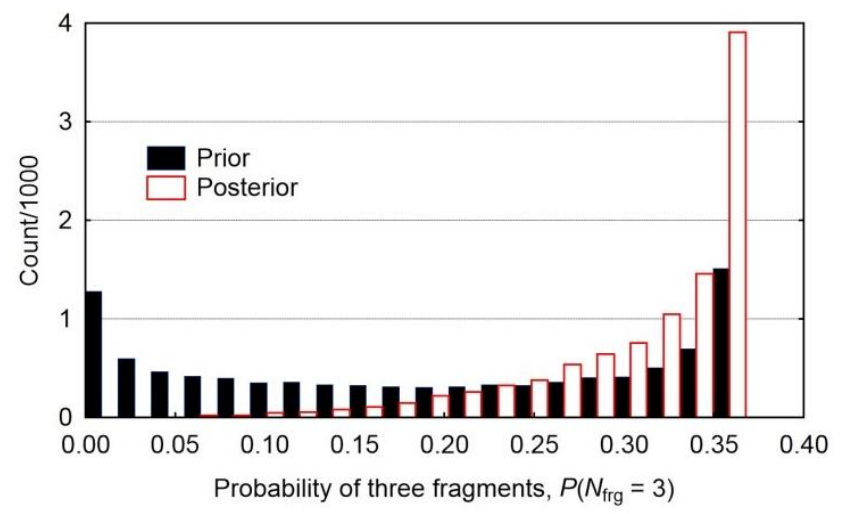

$\mathrm{b}$

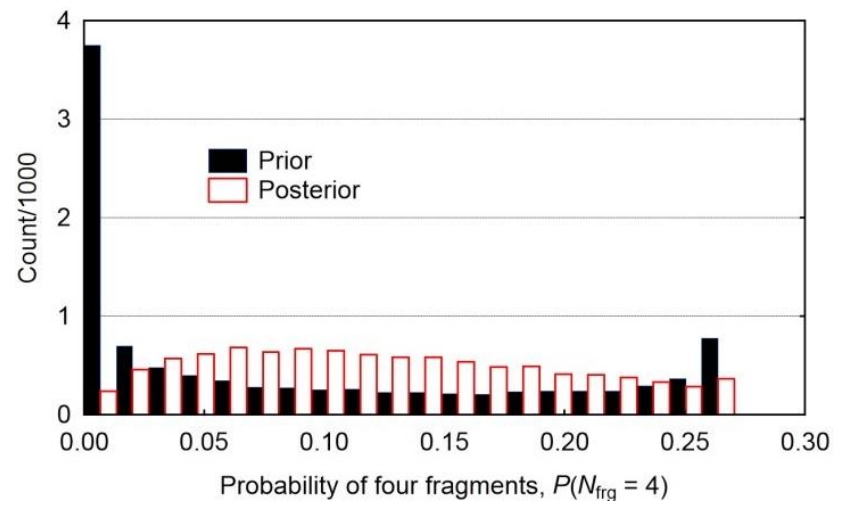

c

Fig. 5 Histograms of the sample pairs $\left(\boldsymbol{p}_{m}, \boldsymbol{p}_{m}{ }_{m}\right)$ generated to assess prior and posterior uncertainty in the fragment number probabilities $P\left(N_{f r g}=m\right):$ a) the case $m=2$; b) the case $m=3$; c) the case $m=4$

The large uncertainty related to the fragmentation probabilities $P\left(N_{f r g}=n_{f r g}\right)$ can be seen as a positive result in a sense. This uncertainty reflects the vagueness of infor- 
mation related to the potential number of fragments generated by vessel explosions. A part of this information is expressed by the data given in Table 2 . This information could not be called very diffuse. For instance, the Clopper-Pearson $95 \%$ confidence intervals of the proportions $f r\left(n_{f r g}\right)$ given in Table 2 are relatively narrow (Table 4$)$. The vagueness related to the probabilities $P\left(N_{f r g}=n_{f r g}\right)$ stems from the decision to use the fairly diffuse CNI prior density of the displaced average number of fragments, $\Lambda_{0}$.

Table 3

Descriptive measures of the samples of probability values, $\boldsymbol{p}_{m}$ and $\boldsymbol{p}_{m}^{\prime}$, generated to assess uncertainty in the probabilities $P\left(N_{f r g}=m\right)(m=2,3,4)$

\begin{tabular}{|c|c|c|c|c|c|}
\hline Sample & Mean & Median & COV, $\%$ & Min & Max \\
\hline $\boldsymbol{p}_{2}$ & 0.624 & 0.697 & 51.4 & $8.6 \times 10^{-6}$ & 1.000 \\
$\boldsymbol{p}_{3}$ & 0.190 & 0.195 & 68.2 & $1.3 \times 10^{-8}$ & 0.368 \\
$\boldsymbol{p}_{4}$ & 0.087 & 0.043 & 109 & $8 \times 10^{-17}$ & 0.271 \\
\hline $\boldsymbol{p}_{2}^{\prime}$ & 0.504 & 0.503 & 34.4 & $4.1 \times 10^{-2}$ & 0.954 \\
$\boldsymbol{p}_{3}^{\prime}$ & 0.314 & 0.336 & 19.2 & $4.5 \times 10^{-2}$ & 0.368 \\
$\boldsymbol{p}_{4}^{\prime}$ & 0.126 & 0.119 & 56.4 & $1.1 \times 10^{-3}$ & 0.271 \\
\hline
\end{tabular}

The decision to use a less diffuse prior distribution of $\Lambda_{0}$ could be difficult to justify in PRA reasoning. A possible argument for this is that the data given in Tables 1 and 2 covers a fairly diverse population of 259 vessels that underwent BLEVEs. It could be difficult to say how many of these vessels are similar or analogous to the specific vessel(s) under study.

Numerical results obtained in this example indicate that the diffuseness of the prior distribution of $\Lambda_{0}$ is not a critical problem due to the influence of new data on the prior. Information on four hypothetical accidents was sufficient to reduce the COV of the distribution of $\Lambda_{0} 2.5$ times, from $141 \%$ to $56 \%$. This led to a tangible reduction of COVs of the samples $\boldsymbol{p}_{m}$ and $\boldsymbol{p}_{m}^{\prime}$, the variability of which indirectly expresses epistemic uncertainty in the fragmentation probabilities $P\left(N_{f r g}=n_{f r g}\right)$.

Table 4

Clopper-Pearson $95 \%$ confidence intervals calculated for three proportions (frequencies) given in Table 2

\begin{tabular}{|c|c|c|}
\hline Proportion & Confidence interval (CI) & Width of CI \\
\hline$f r(2)$ & $(0.400,0.5390)$ & 0.139 \\
$f r(3)$ & $(0.307,0.443)$ & 0.136 \\
$f r(4)$ & $(0.0750,0.1660)$ & 0.091 \\
\hline
\end{tabular}

For a fixed parameter value $\lambda_{0}+2$, the sum of the three probabilities $P\left(N_{f r g}=n_{f r g} \mid \lambda_{0}+2\right) \quad\left(n_{f r g}=2,3,4\right)$ will be a value slightly lower than unity. However, sums of the simulated probability values, $\varepsilon_{i}=\sum_{n_{f r g}=2}^{4} p_{i}\left(n_{f r g}\right)$ and $\varepsilon_{i}^{\prime}=\sum_{n_{f r g}=2}^{4} p_{i}^{\prime}\left(n_{f r g}\right)$, can exceed unity in the case of large variances of the samples $\boldsymbol{p}_{m}$ and $\boldsymbol{p}_{m}^{\prime}$. Descriptive measures and histograms of the simulated samples $\varepsilon=$ $\left\{\varepsilon_{i}, i=1, \ldots, n\right\}$ and $\varepsilon^{\prime}=\left\{\varepsilon_{i}^{\prime}, i=1, \ldots, n\right\}$ are given in Table 5 and Fig. 6.

Table 5

Descriptive measures of the samples $\varepsilon$ and $\varepsilon^{\prime}$ consisting of simulated sums of the prior and posterior fragment number probabilities $p_{i}\left(n_{f r g}\right)$ and $p_{i}^{\prime}\left(n_{f r g}\right)$

\begin{tabular}{|c|c|c|c|c|}
\hline Sample & Mean & $\begin{array}{c}\text { Proportion of ex- } \\
\text { ceedance of 1.0 }\end{array}$ & Min & Max \\
\hline $\boldsymbol{\varepsilon}$ & 0.901 & $0.429(43 \%)$ & $5.9 \times 10^{-4}$ & 1.587 \\
$\boldsymbol{\varepsilon}^{\prime}$ & 0.943 & 0 & 0.382 & 0.99998 \\
\hline
\end{tabular}

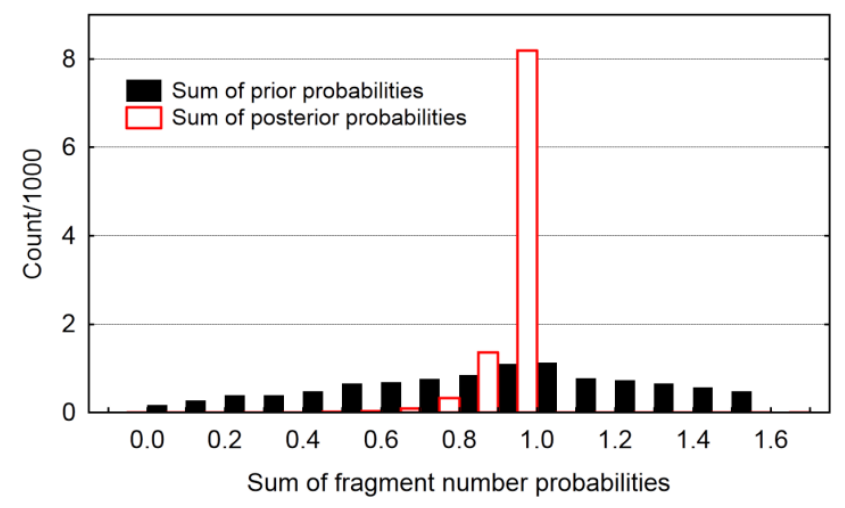

Fig. 6 Histograms of the samples $\varepsilon$ and $\varepsilon^{\prime}$ consisting of simulated sums of the prior and posterior fragment number probabilities $p_{i}\left(n_{f r g}\right)$ and $p_{i}^{\prime}\left(n_{f r g}\right)$

In case of the prior fragment number probabilities $p_{i}\left(n_{f r g}\right), 43 \%$ of elements of the sample $\boldsymbol{\varepsilon}$ exceeded unity with the remainder of elements less than unity (Table 5). In case of the probabilities $p_{i}^{\prime}\left(n_{f r g}\right)$, all elements of $\varepsilon^{\prime}$ were less than unity. This contradicts the fact that the sum of $P\left(N_{f r g}=n_{f r g} \mid \lambda_{0}+2\right) \quad\left(n_{f r g}=2,3,4\right)$ is less than unity, no matter what is the value of Poisson parameter. The problem of statistical variability of $\boldsymbol{\varepsilon}$ and $\boldsymbol{\varepsilon}^{\prime}$ can be sidestepped if the simulated probabilities $p_{i}\left(n_{f r g}\right)$ and $p_{i}^{\prime}\left(n_{f r g}\right)$ are used for a stochastic simulation of explosion accident. The simulation run $i$ must include the decision on how many fragments will be ejected. This decision can be simulated by generating a value of a random variable, $u_{i}$, uniformly distributed over the intervals $\left.] 0, \varepsilon_{i}\right]$ or $\left.] 0, \varepsilon_{i}^{\prime}\right]$.

\section{Discussion}

The Poisson-gamma model applied in this study to an estimation of the fragment number probabilities should be seen only as one of the possibilities to assess accuracy of these probabilities. The estimation was carried out as a propagation of epistemic uncertainty through the probability mass function of the Poisson distribution. The use of this distribution was substantiated by the fact that it fairly well approximates frequencies (proportions) of individual fragment numbers encountered in the past explosion accidents. Empirical frequencies were approximated by interpreting the Poisson parameter lambda as a mean number of fragments per one vessel bursting. The use of this single-parameter model allows a relatively simple Bayesian analysis by 
developing and updating a prior Gamma distribution for lambda.

The Poisson-gamma model has also two disadvantages in the context under study. Firstly, the single-parameter Poisson model may be too rigid for fitting to new data if a distribution of this data will substantially deviate from the standard Poisson scheme. For instance, an abnormally large number of four or five fragments will be counted in future accidents. Secondly, the sum of the fragment number probabilities computed with lambda values sampled from Gamma prior and posterior may exceed unity. This fact violated the axiomatic definition of discrete probability distributions. However, this disadvantage can be easily sidestepped if the generated values of fragment number probabilities are used in an explosion accident simulation to make decision concerning a current number of fragments (see comments at the end of Section 4).

An alternative to the Poisson-gamma model can be the multinomial-Dirichlet model. The former requires to develop only one prior distribution, namely, the distribution of the lambda parameter, However, the latter is more flexible than the former because the multivariate Dirichlet distribution can be used for a direct quantification of epistemic uncertainties in the fragment number probabilities. The uncertainty propagation applied in this study becomes unnecessary. On the other hand, an application of the Dirichlet distribution will require to develop as many priors as many fragments are regarded in the prediction. The aforementioned CNI prior method could be used for this purpose. However, a special study is necessary to answer the question which of the two models is preferable for the prediction of fragment numbers.

\section{Conclusion}

This study proposed a procedure for predicting the number of primary fragments that can be generated by an explosion of a pressure vessel. The prediction in made in the form of fragment number probabilities. Bayesian analysis has been applied to assessing uncertainties related to fragment number probabilities. The Bayesian approach has been used to combine data on number of fragments recorded in the past explosion accidents with expert opinion concerning the average number of fragments per one explosion accident. The main finding is that a Poisson-gamma model can be used for the estimation of fragment number probabilities in the Bayesian format and integrated in the PRA related to pressure vessels.

The current data situation allows to develop a prior distribution of the average number of fragments per accident in the form of a gamma distribution. Epistemic uncertainty related to the average fragment number can be transformed into uncertainty in the fragment number probabilities by means of a stochastic simulation. The proposed procedure can be used for assessing risk posed by potential impact of fragments on vulnerable targets.

The proposed approach was applied to fire induced pressure vessel explosions known as BLEVEs. The procedure of the fragment number assessment is based on the use of post-mortem data on BLEVEs. Unfortunately, the currently available deterministic mechanical modelling does not allow to predict reliably the entire process of vessel fraction that ends up with a formation of circumferential cracks and catastrophic failure of vessel. A convergence of the data-driven prediction and the mechanical modelling, deterministic and probabilistic, is an issue to be solved in the future.

\section{References}

1. Mannan S. (ed.). 2014. Lees's Process Safety Essentials. Hazard Identification, Assessment and Control. Amsterdam: Elsevier. 547 p.

2. Gamberini, L.; Imbriaco, G.; Flauto, A.; et al. 2021. Mass casualty management after a boiling liquid expanding vapor explosion in an urban area, The Journal of Emergency Medicine.

https://doi.org/10.1016/j.jemermed.2020.11.029.

3. Planas, E.; Pastor, E; Casal, J; Bonilla, J. M. 2015. Analysis of the boiling liquid expanding vapor explosion (BLEVE) of a liquefied natural gas road tanker: The Zarzalico accident, Journal of Loss Prevention in Process Industries March: 127-138. http://dx.doi.org/10.1016/j.jlp.2015.01.026.

4. Planas-Cuchi, E.; Gasulla, N.; Ventosa, A.; Casal, J. 2004. Explosion of a road tanker containing liquefied natural gas, Journal of Loss Prevention in Process Industries 17: 315-321. http://doi:10.1016/j.jlp.2004.05.005.

5. Abbasi, T.; Abbasi, S. A. 2007. The boiling liquid expanding vapour explosion (BLEVE): Mechanism, consequence assessment, management, Journal of Hazardous Materials 141(3): 489-519.

http://doi:10.1016/j.jhazmat.2006.09.056.

6. Susan, D. F.; Eckelmeyer, K. H.; Kilgo, A. C. 2005. Metallurgical failure analysis of a propane tank boiling liquid expanding vapor explosion (BLEVE), Journal of Failure Analysis and Prevention 2005 5(5): 65-74. https://doi.org/10.1361/154770205X65918.

7. Iannaccone, T.; Scarponi, G. E.; Landucci, G.; Cozzani, V. 2021. Numerical simulation of LNG tanks exposed to fire, Process Safety and Environmental Protection 149(May): 735-749. https://doi.org/10.1016/j.psep.2021.03.027.

8. Scarponi, G. E.; Landucci, G.; Birk, A. M.; Cozzani, V. 2021. Three dimensional CFD simulation of LPG tanks exposed to partially engulfing pool fires, Process Safety and Environmental Protection. https://doi.org/10.1016/j.psep.2021.04.026.

9. Birk, A. M.; Eyssette, R.; Heymes, F. 2019. Early moments of BLEVE: From vessel opening to liquid flashing release, Process Safety and Environmental Protection 132(December): 35-46. https://doi.org/10.1016/j.psep.2019.09.028.

10. Bubbico, R.; Bigg, P.; Mazzarotta, B. 2019. Protection systems for tanks containing hazardous materials exposed to fire, Chemical Engineering Transactions 77: 229-234. https://doi:10.3303/CET1977039.

11. Pula, R.; Khan, F.I.; Veitch B.; Amyotte, P. R. 2006. A model for estimating the probability of missile impact: missiles originating from bursting horizontal cylindrical vessels. Process Safety Progress 26(2): 129-139. https://doi.org/10.1002/prs.10178.

12. Vaidogas, E. R. 2021. Predicting the ejection velocities of fragments from explosions cylindrical pressure vessels: Uncertainty and sensitivity analysis. Journal of Loss Prevention in the Process Industries. 
https://doi.org/10.1016/j.jlp.2021.104450.

13. Li, Z.; Sun, D.; Sun, J.; Jiang, J. 2021. Study on the number of primary and secondary fragments produced by explosion of horizontal vessel, Lecture Notes in Civil Engineering 110, 399-407. https://doi.org/10.1007/978-981-15-9121-1_28.

14. Sun, D.; Jiang, J.; Zhang, M.; Wang, Z.; Huang, G.; Qiao, J. 2012. Parametric approach of the domino effect for structural fragments, Journal of Loss Prevention in the Process Industries 25(1): 114-126. http://doi:10.1016/j.jlp.2011.06.029.

15. Mébarki, A.; Mercier, F.; Nguyen, Q. B; Ami Saada, R. 2009. Structural fragments and explosions in industrial facilities. Part I: Probabilistic description of the source terms, Journal of Loss Prevention in the Process Industries 22(4): 408-416. http://doi:10.1016/j.jlp.2009.02.006.

16. Juocevicius, V.; Vaidogas, E. R. 2010. Effect of explosive loading on mechanical properties of concrete and reinforcing steel: towards developing a predictive model, Mechanika 81(1): 5-12.

17. Kelly, D.; Smith, C. 2011. Bayesian Inference for Probabilistic Risk Assessment: A Practitioner's Guidebook. London: Springer.

18. Kelly, D.; Atwood, C. 2011. Finding a minimally informative Dirichlet prior distribution using least squares, Reliability Engineering \& System Safety 96(3): 398402. http://doi:10.1016/j.ress.2010.11.008.

19. Atwood, C. L. 1996. Constrained noninformative priors in risk assessment. Reliability Engineering \& System Safety 53:37-46. https://doi.org/10.1016/0951-8320(96)00026-9.
E. R. Vaidogas

\section{EXPLOSIONS OF CYLINDRICAL PRESSURE VESSELS SUBJECTED TO FIRE: PROBABILISTIC PREDICTION OF A NUMBER OF FRAGMENTS}

\section{S u m m a r y}

The aim of this study was to propose a procedure for a prediction of the number of fragments generated by fire induced explosions of cylindrical pressure vessels. The prediction is carried out in terms of probabilities of individual fragment numbers. The prevailing numbers of two to four fragments are considered. The fragment number probabilities are estimated by applying data on vessel fragmentations acquired in investigations of past explosion accidents. The pressure vessel explosions known as BLEVEs are considered. The Bayesian analysis is used for the estimation of the fragment number probabilities. This analysis is carried out on the basis of Poisson-gamma model. An approach to developing a gamma prior distribution for the average number of fragments per explosion accident is proposed. The assessment of the fragment number probabilities is carried out by propagating uncertainty related to the average number of fragments to uncertainty in the fragment number probabilities. The stochastic (Monte Carlo) simulation is used for this propagation. Findings of this study are viewed as a possibility to improve the assessment of risk posed by pressure vessel explosions.

Keywords: pressure vessel, explosion, BLEVE, fragment, Bayesian analysis, epistemic uncertainty, Poisson-gamma model. 